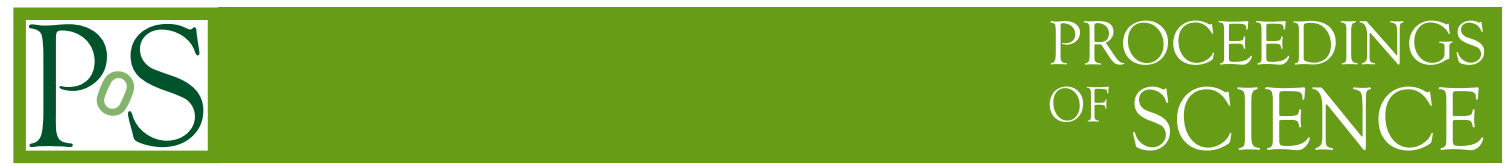

\title{
Theoretical constraints on modified theories of gravity
}

\section{Keisuke Izumi*}

KMI, Dept. of Math., Nagoya U.

E-mail: izumi@math.nagoya-u.ac.jp

\begin{abstract}
We discuss about the theoretical constraints on modified theories of gravity. To explain the dark component of the Universe, many modified theories of gravity have been proposed. Meanwhile, to quantize the gravitational theory, we would need a new theory of gravity in UV physics. In this talk, we focus on three theoretical aspects of gravitational theories; causal structures, positivity of energy and black hole physics. The recent developments of these topics were discussed.
\end{abstract}

The 3rd International Symposium on "Quest for the Origin of Particles and the Universe" 5-7 January 2017

Nagoya University, Japan

${ }^{*}$ Speaker. 


\section{Introduction}

To explain the effects of dark components of the Universe, modified theories of gravity are discussed and/or exotic matters are introduced. On the other hand, to construct the unified quantum theory, gravitational theories beyond Einstein gravity are discussed.

Einstein theory has good mathematical structures; good causal structure, positivity of theory and theorems about black holes. These mathematical theorems are important not only for the beauty (i.e. simplicity) but also for physics to be well defined. We discuss about these mathematical structures in various modified theories of gravity.

\section{Causal Structure}

The causal structure of general relativity with usual matters can be discussed with null curves. Here, usual matters mean that their kinetic terms have the canonical form. The reason why the discussion is based on null curves is that the speed of all propagations is slower than or equal to that of light, that corresponds to null direction.

To explain the dark components of the Universe, many modified theories of gravity have been proposed. The exotic matter (such as Galileon field) is also considered for this purpose. Many modified theories of gravity have often superluminal propagation. The exotic matter sometimes propagates superluminally. Meanwhile, to quantize gravitational theory, we consider the gravity beyond the Einstein theory. Such a theory is called quantum gravity. The major candidate of quantum gravity theory is the superstring theory. The superstring theory is reduce to the Einstein theory in the infrared limit, i.e. in the low-energy limit the action has the linear of curvature. When we take the next leading order into account, the higher-order curvature terms would appear in the action. This higher-order curvature terms make the propagation of gravitons superluminal. Therefore, modified theories of gravity from the Einstein's general relativity (and also theories with some exotic matters) potentially have superluminal propagation.

If superluminal propagations are included in the theory, the causal analysis based on null curve is meaningless. The causal analysis with null curve can be justified only with the following assumption; the speed of the fastest propagation is the same as that of light. The existence of the superluminal modes manifestly breaks this assumption. Therefore, the causal structure becomes non-trivial.

Superluminal propagations sometimes break the causal structure. In the usual theory (i.e. general relativity with usual matters), local acausality does not happen. This is because the concept of light cone divides a local patch into future and past (and spacelike) regions. However, in theories with superluminal modes we sometimes do not have this concept. If we have similar cone corresponding to the fastest propagation, we indeed can discuss the future and past. Nevertheless, most of modified gravity theories with superluminal modes, the cone is broken in some configuration of fields. Then, we can not define future and past, and the theories are acausal. We stress that here the acausality is local, not global. Actually, we can construct the solution with global acausality in general relativity. The trivial solution is Minkowski solution with the compactification in time direction, say we identify $t=0$ hypersurface as $t=1$ hypersurface. This is nonsense, that is we just take a bad solution. If we start from the initial condition on $t=0$ hypersurface, the 
time evolution gives global Minkowski solution without the compactification. In this sense, the global acausality is an artificially product. In time evolution problem, we can choose the solution without the global acausality. However, the local acausality is pathological. The local acausality is dynamically created and we have no way to solve the time evolution beyond it.

Checking this acausal structure, we can evaluate the consistency of the modified theories of gravity. In a torsion gravity called $f(T)$ gravity, the local acausality appears even in the Minkowski vacuum and Friedmann background $[4,5,6]$. This means that this theory is pathological even in low-energy limit, and thus it is rejected. In massive gravity, we can construct a solution on which the local acausality appears $[7,8,9,10]$. Therefore, to save the theory, such solutions are out of the region where the theory can be apply as an effective field theory. Thus, this theory is never the fundamental theory. The cut-off energy scale should be carefully checked.

Another nontrivial and interesting object related to causality is black hole. Black holes are defined as a region where the information can not reach the future null infinity. With superluminal modes, the black hole horizon (corresponding to the boundary of the region where the all observers at infinity can never see) does not need to be null hypersurface. Nevertheless, in many works, black holes in the theory with superluminal modes are discussed based on null curves. In such theories, the analysis should be done with the fastest modes.

In this talk, we show the causal structure in Gauss-Bonnet-Einstein gravity [11] (see also Ref.[12,13]). Because of higher order curvature term, graviton can propagate superluminally on some configuration of fields. However, the Killing horizon, that is a null surface, becomes the boundary of the causal structure, that is, the graviton propagates have the same speed as light on the Killing horizon.

On dynamical black holes, the situation is different. Especially on shrinking black holes (by Hawking radiation), the causal structure becomes nontrivial. The graviton propagating to outward direction becomes superluminal on the "horizon" defined by null curves in shrinking black holes. Therefore, the "horizon" defined by null curves is not the causal boundary. The horizon, that is the causal boundary, is inside the "horizon" defined by null curves. The classical waves can escape from the "black hole" defined by null curves.

\section{Positivity of energy}

Positivity of energy is important concept in physics. If we have negative energy excitations in a vacuum, the vacuum is unstable. Even if the vacuum has only positive energy excitations perturbatively, if the Hamiltonian is not bounded from below in nonlinear regime, the system is unstable by quantum effect. Thus, the positivity of energy is required in well defined physics.

The positivity of energy in general relativity was firstly proven by Schoen and Yau [14]. The proof was done by the minimal slice deformation. After Schoen and Yau, Witten's proof in different way appeared $[15,16]$. Witten's proof is based on the supersymmetry, and thus many extensions can be discussed. [17, 18, 19, 20] For instance, Bogomol'nyi-Prasad-Sommerfield bound can be derived by the extension of Witten's proof [20].

In cosmology, many theories of scalar field are discussed, for instance, k-essense which is the theory with nontrivial kinetic structure. Based on Witten's proof, the theory having positive energy 
is recently discussed $[21,22,23]$. Only if the theory has the canonical kinetic structure, Hamiltonian is proven to be bounded from below. Therefore, noncanonical kinetic term is unfavored.

\section{Black hole physics}

In general relativity, we have theorems about black holes. Uniqueness theorem would show the final equilibrium state of black hole spacetime. Penrose inequality give the maximum value of the area of black hole horizon. We can check the validity of general relativity based on these theorems. However, these theorems are proven with the assumption of the existence of black hole horizons. The black hole horizon can not be observed by definition. Thus, these theorems are discussed by the variables that we can never observe.

Recently, these theorems not based on the existence of black hole horizon are discussed. To discuss these theorems, we perhaps need a strong gravity region. Therefore, we need a region where gravity is enough strong but not too strong for black hole horizons to exist. One of candidates describing such a strong gravity region is photon surface. In Schwarzschild spacetime, we have circular orbits of photon. These circular orbits create a surface, which is called photon surface (or photon sphere if it is sphere). This trapping photon on the circular orbit happens because of strong gravity and exists outside horizon.

We briefly show the recent discussions of the uniqueness theorem and Penrose inequality based on the photon surface.

\subsection{Uniqueness theorem}

The Uniqueness theorem is a strong theorem in black hole physics. The theorem says that the stationary black holes are characterized only three parameters; mass, angular momentum and electric charge. Suppose dynamics is settled into an equilibrium state. Then, the final equilibrium state of black hole is fixed only by mass, angular momentum and electric charge. The strong point of this theorem is that not only the black hole itself but also the whole spacetime structure is determined. Therefore, only with the observation of mass, angular momentum and electric charge, we can know the whole spacetime structure.

Recently, the uniqueness theorem for photon surface is discussed [24, 25, 26, 27, 28, 29, 30]. Its motivation is, as we commented, discussing the theorem with the obeservable quantity. As a first step, static cases are mostly discussed.

The main difference is that photon surface has less properties than black hole horizon. Because of less properties, it hard to prove the uniqueness only with the assumption of the existence of photon surface. Assuming the constancy of the lapse function on photon surface, it is possible to prove the uniqueness of photon surface. It is enough interesting but the proof with less assumption is desired. Moreover, the uniqueness theorem in stationary cases is important future direction.

In a special case, Einstein theory with conformal scalar field, the uniqueness of photon surface can be proven without the assumption of the constancy of the lapse function on photon surface $[31,32]$. In this proof, we do not even the existence of photon surface a priori. It is possible to show that, if there is a scalar hair, there is a photon surface and the spacetime outside photon surface is uniquely fixed. 


\subsection{Penrose inequality}

While the uniqueness theorem is important to discuss a stationary black hole, Penrose inequality can be applied even in highly dynamical case. Penrose inequality says that the area $A$ of black has upper bound determined by ADM energy $m$;

$$
A \leq 4 \pi(2 G m)^{2} .
$$

The inequality is enough interesting but for horizon that can never be observed by definition. We modified the inequality for a strong gravity region named loosely trapped surface, that we define as follows [33].

Definition: The loosely trapped surface, $S_{0}$, is defined as a compact 2-surface such that its trace of the extrinsic curvature $k$ (for the outward spatial direction) is positive $\left(\left.k\right|_{S_{0}}=: k_{0}>0\right)$ and the derivative along the outward spatial direction is non-negative $\left(\left.k^{\prime}\right|_{S_{0}} \geq 0\right)$ in a spacelike hypersurface $\Sigma$.

This definition can be applied for arbitrary initial data with the induced metric and extrinsic curvature $\left(q_{a b}, K_{a b}\right)$ satisfying the Hamiltonian and momentum constraints. Then, unlike the Schwarzschild spacetime, a loosely trapped surface is not necessarily located outside of an apparent horizon in general. But, it is still a useful indicator for a strong gravity region. Then, we have an inequality for loosely trapped surface;

Theorem: Let $\Sigma$ be an asymptotically flat spacelike hypersurface with non-negative Ricci scalar

${ }^{(3)} R$ and assume that $\Sigma$ is foliated by the inverse mean curvature flow $\left\{S_{y}\right\}_{y \in \mathbf{R}}\left(S_{y} \approx S^{2}\right)$. Then, the area $A_{0}$ of a loosely trapped surface $S_{0}$ in $\Sigma$ satisfies the inequality

$$
A_{0} \leq 4 \pi(3 G m)^{2}
$$

where $m$ is the ADM mass. When the equality holds, the region $\Omega$ outside of $S_{0}$ in $\Sigma$ is isometric to the region of $\Omega_{\text {sch }}$ outside of the photon sphere in the $t=$ const. slice of the Schwarzschild spacetime.

\section{References}

[1] Y. Tomikawa, T. Shiromizu and K. Izumi, arXiv:1702.05682 [gr-qc].

[2] T. Shiromizu, Y. Tomikawa, K. Izumi and H. Yoshino, arXiv:1701.00564 [gr-qc].

[3] Y. Tomikawa, T. Shiromizu and K. Izumi, arXiv:1612.01228 [gr-qc].

[4] P. Chen, K. Izumi, J. M. Nester and Y. C. Ong, Phys. Rev. D 91, no. 6, 064003 (2015) [arXiv: 1412.8383 [gr-qc]].

[5] K. Izumi, J. A. Gu and Y. C. Ong, Phys. Rev. D 89, no. 8, 084025 (2014) [arXiv:1309.6461 [gr-qc]].

[6] Y. C. Ong, K. Izumi, J. M. Nester and P. Chen, Phys. Rev. D 88, 024019 (2013) [arXiv:1303.0993 [gr-qc]].

[7] S. Deser, K. Izumi, Y. C. Ong and A. Waldron, Mod. Phys. Lett. A 30, 1540006 (2015) [arXiv:1410.2289 [hep-th]].

[8] S. Deser, K. Izumi, Y. C. Ong and A. Waldron, arXiv:1312.1115 [hep-th]. 
[9] S. Deser, K. Izumi, Y. C. Ong and A. Waldron, Phys. Lett. B 726, 544 (2013) [arXiv:1306.5457 [hep-th]].

[10] K. Izumi and Y. C. Ong, Class. Quant. Grav. 30, 184008 (2013) [arXiv:1304.0211 [hep-th]].

[11] K. Izumi, Phys. Rev. D 90, no. 4, 044037 (2014) [arXiv:1406.0677 [gr-qc]].

[12] H. Reall, N. Tanahashi and B. Way, Class. Quant. Grav. 31, 205005 (2014) [arXiv:1406.3379 [hep-th]].

[13] M. Minamitsuji, Phys. Lett. B 743, 272 (2015).

[14] R. Schoen and S. T. Yau, Commun. Math. Phys. 79, 231 (1981).

[15] E. Witten, Commun. Math. Phys. 80, 381 (1981).

[16] J. A. Nester, Phys. Lett. A 83, 241 (1981).

[17] P. K. Townsend, Phys. Lett. 148B, 55 (1984).

[18] G. W. Gibbons, C. M. Hull and N. P. Warner, Nucl. Phys. B 218, 173 (1983).

[19] T. Parker and C. H. Taubes, Commun. Math. Phys. 84, 223 (1982).

[20] G. W. Gibbons and C. M. Hull, Phys. Lett. 109B, 190 (1982).

[21] M. Nozawa and T. Shiromizu, Phys. Rev. D 89, no. 2, 023011 (2014) [arXiv:1310.1663 [gr-qc]].

[22] M. Nozawa and T. Shiromizu, Nucl. Phys. B 887, 380 (2014) [arXiv:1407.3355 [hep-th]].

[23] B. Elder, A. Joyce, J. Khoury and A. J. Tolley, Phys. Rev. D 91, no. 6, 064002 (2015) [arXiv:1405.7696 [hep-th]].

[24] S. S. Yazadjiev, Phys. Rev. D 91, no. 12, 123013 (2015) [arXiv:1501.06837 [gr-qc]].

[25] C. Cederbaum, arXiv:1406.5475 [math.DG].

[26] C. Cederbaum and G. J. Galloway, arXiv:1504.05804 [math.DG].

[27] C. Cederbaum and G. J. Galloway, Class. Quant. Grav. 33, 075006 (2016) [arXiv:1508.00355 [math.DG]].

[28] S. Yazadjiev and B. Lazov, Class. Quant. Grav. 32, 165021 (2015) [arXiv:1503.06828 [gr-qc]].

[29] M. Rogatko, Phys. Rev. D 93, no. 6, 064003 (2016) [arXiv:1602.03270 [hep-th]].

[30] H. Yoshino, Phys. Rev. D 95, no. 4, 044047 (2017) [arXiv:1607.07133 [gr-qc]].

[31] Y. Tomikawa, T. Shiromizu and K. Izumi, arXiv:1702.05682 [gr-qc].

[32] Y. Tomikawa, T. Shiromizu and K. Izumi, arXiv:1612.01228 [gr-qc].

[33] T. Shiromizu, Y. Tomikawa, K. Izumi and H. Yoshino, arXiv:1701.00564 [gr-qc]. 